\title{
MicroscopyPioneers
}

\section{Cryosectioning and Immunolabeling: The Contributions of Kiyoteru Tokuyasu}

\author{
Gareth Griffiths, ${ }^{1}$ Jan-Willem Slot, ${ }^{2}$ and Paul Webster ${ }^{3 *}$ \\ ${ }^{1}$ Dept of Biosciences, University of Oslo, 0316 Oslo, Norway \\ ${ }^{2}$ Dept of Cell Biology, University Medical Center Utrecht, Utrecht, The Netherlands \\ ${ }^{3}$ Oak Crest Institute of Science, 132 W Chestnut Ave., Monrovia, CA
}

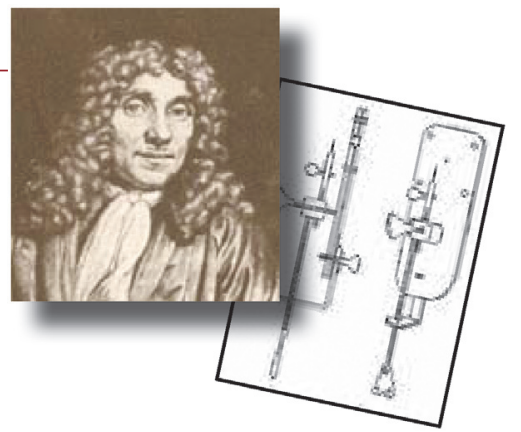

*p.webster@oak-crest.org

Today it is taken for granted that ultrathin sections of frozen biological material can be cut, labeled, and observed in a transmission electron microscope (TEM), but to do this successfully for the first time was a totally different story. Below is a short description of how Kiyoteru Tokuyasu (1925-2015) working in San Diego in the 1970s developed the technique for preparing cryosections of fixed and cryoprotected biological material.

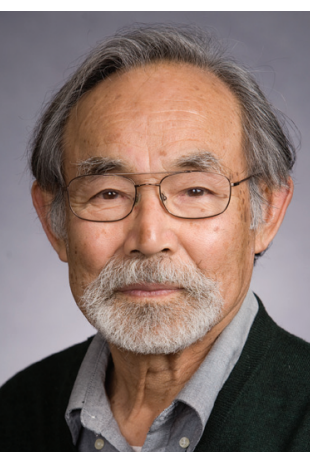

Professor Kiyoteru Tokuyasu
By 1973, the conventional method for ultrastructural analysis was well established. Glutaraldehydecrosslinked biological material could be contrasted with osmium tetroxide, dehydrated, and embedded in resins for thin sectioning. This approach, while still widely used for ultrastructure today, is chemically too harsh for preserving antigens if they are to be detected on the sections with specific antibodies. Cutting sections of frozen material was already theoretically attractive by the 1960s as a gentler alternative to resins. In fact, Fernandes-Moran had experimented with sectioning unembedded frozen material as early as 1952 [1]. He was able to produce sections thin enough for examination in the TEM using a modified histology microtome in a $-35^{\circ} \mathrm{C}$ cold room. Poor morphology, together with the introduction of methacrylate resins, led to cryosectioning being temporarily abandoned. It was Tokuyasu who found practical solutions for several key steps of the method that now bears his name [2] and has made the technique of using thawed cryosectioning such a key technique for cell biology for the last 40 years.

There were, and still are, two main reasons to consider cutting thin sections of frozen cells or tissues: first to analyze their structure, and second to label sections with antibodies and other reagents after thawing the sections. The former approach was developed by Jacques Dubochet and colleagues and is now known as cryo EM of vitrified sections (CEMOVIS). A key conceptual breakthrough for this method to work was to understand the process of vitrification, the ability to solidify water so fast that ice crystals cannot form [3]. Dubochet was awarded the Nobel Prize in Chemistry for this work in 2017, a prize shared with Joachim Frank and Richard Henderson.
The CEMOVIS method is justifiably referred to as "cryo EM" because the sections are observed in the vitreous state. In contrast, transiently freezing a tissue in order to obtain sections that are subsequently thawed for labeling should not be referred to as cryo EM; nevertheless, the principle of vitrification is important also for the latter approach because it turns out that the specimen must be vitreous to allow it to be sectioned in a useful way. Crystalline ice tends to crumble rather than cut smoothly into sections.

The concept of vitrifying water was not known until 1980 $[3,4]$, and even today vitrification of native specimens is a challenging task. What Tokuyasu discovered serendipitously was that by immersing aldehyde-fixed cells or tissues in a high concentration of sucrose, the material could be frozen in liquid nitrogen in such a way that it was easy to section at temperatures near $-100^{\circ} \mathrm{C}$ in a cryo-ultramicrotome. Although he was not aware of the vitrification concept per se, he could always see that "well-frozen" samples of most specimens were as transparent as glass. It was later shown that the key role of sucrose was indeed to enable vitrification, even of large samples, and with slow cooling [5]. Raising the sucrose solution to near saturation (2.1-2.3 M) by the Utrecht group was an important step in the later development of the Tokuyasu technique to a routine method, which is still one of the best and most efficient methods for immunolocalization studies [6].

Another fortuitous property of sucrose-infused cryo specimens is that they have the necessary plasticity to allow ribbons of adjacent thin sections to be cut, a key factor in allowing the Tokuyasu method to develop into a routine method. Earlier developments, especially by Wilhelm Bernard and colleagues in Paris, came close to success, using, for example, glycerol rather than sucrose, but the quality of the images that Tokuyasu showed in his 1973 paper [2] far exceeded any of the preceding publications.

Another key innovation in this 1973 paper was the dry cutting of sections. This avoided chemicals such as DMSO to stretch the sections and allowed the pick up of dry sections from the glass knife (the improved preparation of which was another key Tokuyasu invention) [7]. Using a cryosectioning bowl designed by A. Kent Christensen (Figure 1), fitted to a Sorvall MT-2 ultramicrotome [8, 9], Tokuyasu was able to obtain thin sections on a dry knife, picking them up using a drop of $2.3 \mathrm{M}$ sucrose on an eyelash to fish out and stretch them before transfer to EM grids. Later he replaced this method using 


\section{Kammrath Weiss}


1 to 50 MEGAPIXELS live and slow scan

MAGNIFICATION FACTOR OF 1 on bottom mounted cameras

DIFFRACTION BEAM STOP on side mounted cameras


Affordable TEM camera systems for research, education, healthcare, and industry since 2001

Scientific Instruments and Applications 2773 Heath Lane • Duluth, GA 30096 (770) 2327785 • www.sia-cam.com 


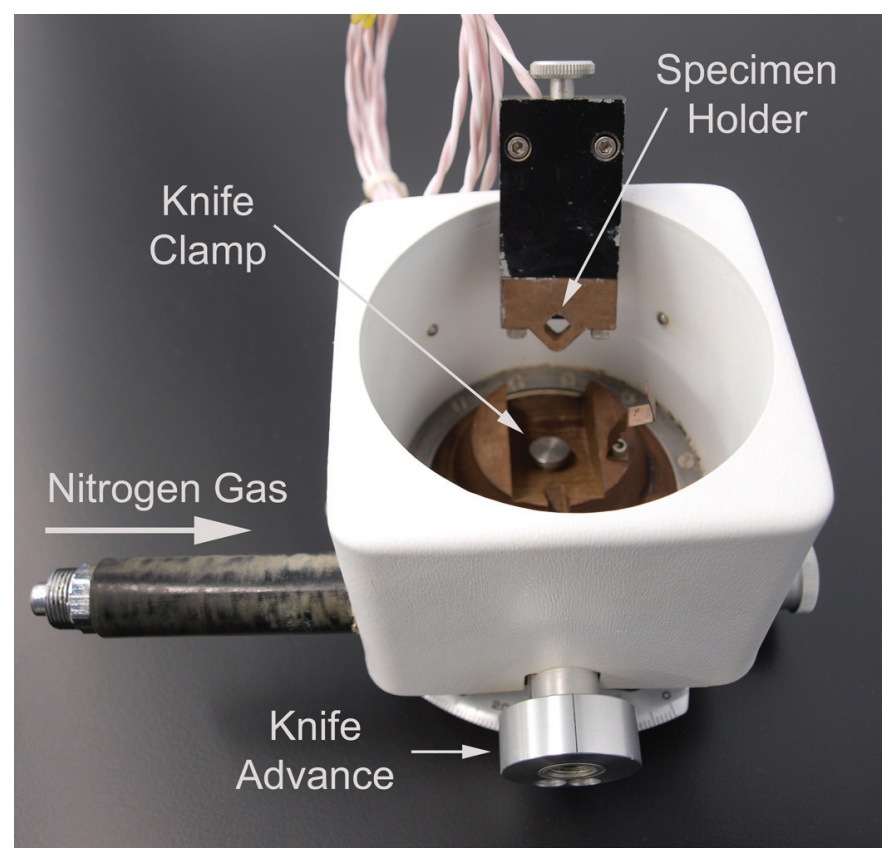

A modified Sorvall cryo-unit, designed by A. Kent Christensen, similar to the one used by Tokuyasu to obtain his cryosections for labeling. The cryo-chamber was cooled by a jet of nitrogen gas, pumped in by heating liquid nitrogen in a sealed Dewar. When a low temperature was reached, chemically crosslinked tissues cryoprotected in sucrose were frozen onto copper stubs and clamped onto the specimen holder. The holder was suspended in the cryo-chamber and attached to a bridge, which came over the top of the chamber to attach to the specimen arm of the ultramicrotome. A knife was clamped onto the ultramicrotome with a knife clamp fastened to the base of the chamber. The knife could be advanced to the frozen specimen using the knife advance on the front of the chamber. Once the specimen and knife were aligned, sectioning could be performed using the ultramicrotome controls.

a wire loop filled with sucrose to pick up the sections from the dry knife-an approach that is still used today.

After picking up the sections, Tokuyasu faced another problem: how to produce contrast in sections while avoiding the serious problem of air-drying before introducing the grids into the vacuum of the electron microscope. His solution was to embed the sections in a relatively thick layer containing heavy metals, such as uranyl acetate, in effect (mostly) negatively staining the sections. The wide range of cells and tissues that he showed as examples in this 1973 paper stand out as excellent examples of ultrastructure [2], as well as of aesthetics; many examples of EM shown today do not reach this level.

However, Tokuyasu then faced another problem. His main goal in developing his technique was to apply it for immunolocalization of antigens, a long passion of his boss, Jon Singer in San Diego. Singer's group was the first to apply antibodies coupled to ferritin as an electron-dense tag that could be seen by EM, using (poorly preserved) specimens embedded in bovine serum albumin [10]. The problem was that ferritin is not very electron opaque. The much denser colloidal gold was only just being introduced to electron microscopy [11] with nanoparticle-sized immunogold under $30 \mathrm{~nm}$ being visualized in 1975 [12]. The result of using a low-density marker was that the denser layer of heavy metal stain, which Tokuyasu needed to support the cryosections during drying, obscured the ferritin label. When the method was applied for the first time in combination with antibody labeling and ferritin [13],

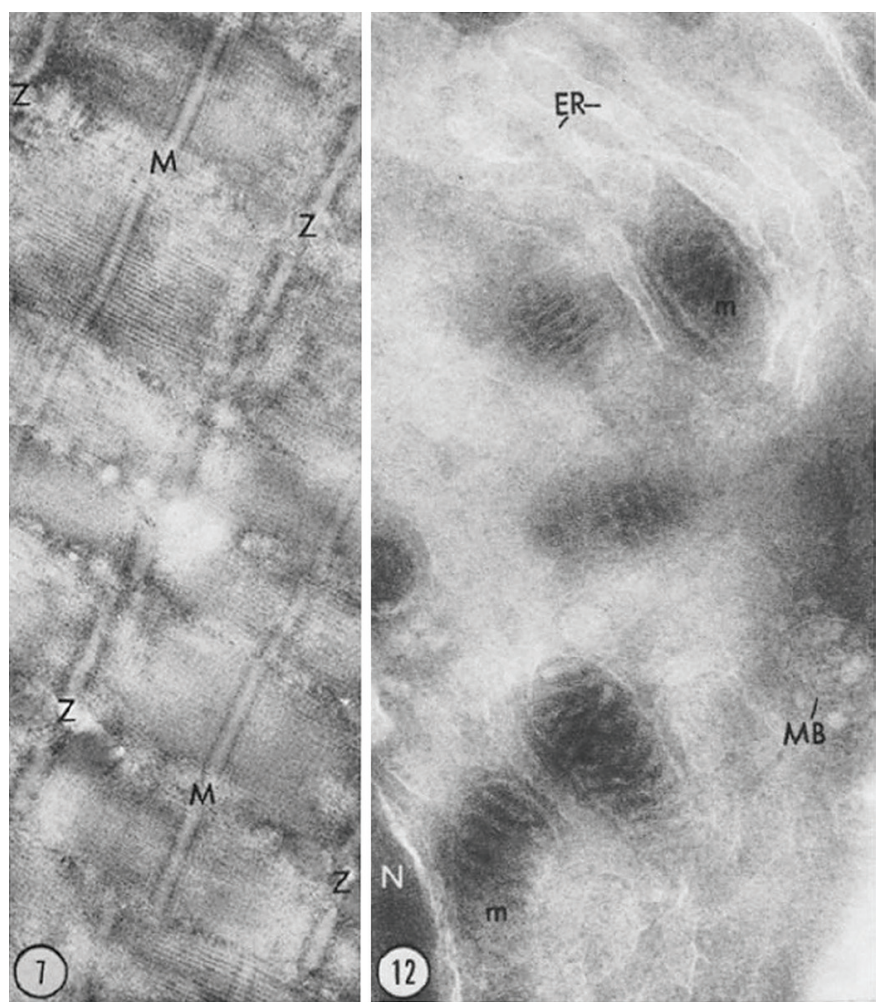

Two figures from Tokuyasu's original 1973 paper in the Journal of Cell Biology [2]. Tissues were fixed in $2 \%$ glutaraldehyde in $100 \mathrm{mM}$ phosphate buffer for up to $1 \mathrm{hr}$, infused with $0.6 \mathrm{M}$ sucrose, and frozen onto a copper holder by immersion in liquid nitrogen. Sections were obtained at $-70^{\circ} \mathrm{C}$ using either glass or diamond knives, and the ultramicrotome was cranked by hand. (left) A section of rat skeletal muscle negatively stained with $0.5 \%$ aqueous uranyl acetate. Image width approx. $2.5 \mu \mathrm{m}$. (right) Part of a plasma cell from mouse spleen. Mitochondria $(\mathrm{m})$ are clearly visible in the negatively stained section. The low contrast reveals endoplasmic reticulum (ER), a multivesicular body (MB), and part of a nucleus $(\mathrm{N})$. The remarkable preservation of mitochondria attracted the attention of microscopists because it was difficult to preserve these structures in resin-embedded sections. Image width approx. $2.8 \mu \mathrm{m}$.

the results were structurally disappointing since the preservation was a compromise between using enough uranyl acetate for contrast and to give some structural preservation, but not enough to obscure the ferritin.

The solution came five years later in a second landmark paper [14] in which Tokuyasu described his new trick of embedding the sections on the grids, not with pure uranyl acetate but with a mixture of (a low concentration) of uranyl acetate and $2 \%$ methyl cellulose to provide a relatively thick scaffold that dried to a layer thin enough for electrons to easily penetrate. This allowed both excellent structural preservation and clear visualization of ferritin (that was soon to be replaced by colloidal gold). For this embedding step Tokuyasu introduced a wire loop, that has remained an integral part of the Tokuyasu technique, and which is used today in hundreds of labs all over the world. It is also necessary to mention the use of the Tokuyasu method for immunofluorescence microscopy, an approach first published by Bourguigon et al. [15].

Over the past 35 years there has been continuous improvement in ultramicrotomes, diamond knives, and in various technical modifications used, such as the solutions for section pick-up, etc. This has turned a demanding, unpredictable 
method into a routine technique $[16,17]$ that can now be quickly learned and applied to a wide range of biological applications. Nevertheless, the foundations of this success were the two beautiful papers by Tokuyasu in 1973 and 1978 [2, 14].

\section{Kiyoteru Tokuyasu}

Affectionately called Tok, he was born in Nagasaki, Japan, in 1925. He studied physics (with a degree 1949) and completed a $\mathrm{PhD}$ in medical sciences at Kyushu University in 1957. His PhD work focused on ultrastructure of the cat retina [18] (Tokuyasu and Yamada 1959). After two years as associate professor in the electron microscopy lab of Kurume Medical College, he was hired as head EM designer for Hitachi (1958-1963). He moved to the USA in 1964 as associate research biologist in the Department of Pathology at UCLA. In 1969 he was attracted to UCSD by Jon Singer, who had the vision to realize that the cryo-sectioning method had great potential for immunolabeling and the even greater vision to give Tok the support he needed to create the Tokuyasu method. Tok was a regular and welcome visitor to Europe. He was for many years an instructor in the annual European Molecular Biology Organization (EMBO) practical course. It is remarkable to note that the first course organized in 1979, at the European Molecular Biology Laboratory in Heidelberg, Germany, focused exclusively on the Tokuyasu method described in an article that was published only a year earlier! This was, in retrospect, highly ambitious because in those days the ultramicrotomes were rudimentary, and the technique required much patience. Today, the technique can be rapidly learned and is taught in several specialized courses. Prof. Tokuyasu was awarded the MSA Distinguished Scientist Award in 2000 .

\section{Courses that Teach the Tokuyasu Cryosectioning Method}

The fastest way to learn about immunocytochemistry and how to prepare thawed cryosections for immunolabeling is to attend a course. These courses are offered occasionally by various organizations. The European Molecular Biology Organization (EMBO) sponsors an annual "Advanced Electron Microscopy for Cell Biology" practical course. The course teaches the Tokuyasu technique for immunolabeling as well as covering all aspects of immunocytochemistry for electron microscopy and other important EM specimen preparation methods for biologists. In 2018 the course was held at the University of Würzburg, Germany. In 2019 the course will be held at the University of South Bohemia in the Czech Republic. The Department of Cell Biology at the University of Utrecht holds basic and advanced courses for sectioning and immunolabeling (http://www.cellbiology-utrecht.nl/em-courses.html). Courses held in response to demand are also organized by Marine Reef International (http://www.marinereef.com/courses.php) and RMC-Boeckeler (http://www.rmcboeckeler.com). Electron Microscopy Sciences include cryosectioning of fixed frozen biological material in their academy curriculum (https://www. emsdiasum.com/microscopy/academy/courses/courses.aspx). Cryoultramicrotomes and information about them can be obtained from Leica Microsystems and RMC-Boeckeler.

\section{References}

[1] H Fernandez-Moran and AO Dahl, Science (1952) 116(3018) 465-67.

[2] K Tokuyasu, J Cell Biol 57(2) (1973) 551-65.

[3] J Dubochet and A McDowall, J Microsc Oxford 124(3) (1981) 3-4.

[4] P Brüggeller and E Mayer, Nature 288(5791) (1980) 569-71.

[5] G Griffiths et al., J Ultra Mol Struct R 89(1) (1984) 65-78.

[6] JW Slot and HJ Geuze, Nat Protoc 2(10) (2007) 2480-91.

[7] K Tokuyasu and S Okamura, J Biophys Biochem Cy (1985) 305-08.

[8] A Christensen, A way to prepare frozen thin sections of fresh tissue for electron microscopy and in Autoradiography of Diffusible Substances eds LJ Roth and WE Stumpf, Academic Press, London, New York, 1969, pp. 349-62.

[9] AK Christensen, J Cell Biol 51(3) (1971) 772-804.

[10] J McLean and S Singer, P Natl Acad Sci 65(1) (1970) 122-28.

[11] WP Faulk and GM Taylor, Immunochemistry 8(11) (1971) 1081-83.

[12] MJ Horisberger et al., Experientia 31(10) (1975) 1147-49.

[13] K Tokuyasu and S Singer, J Cell Biol 71(3) (1976) 894-906.

[14] K Tokuyasu, J Ultra Mol Struct R 63(3) (1978) 287-307.

[15] LY Bourguignon et al., J Cell Physiol 95(3) 1978) 239-57.

[16] E Bos et al., J Struct Biol 175(1) (2011) 62-72.

[17] P Webster and A Webster, "Cryosectioning Fixed and Cryoprotected Biological Material for Immunocytochemistry," in Electron Microscopy, ed J Kuo, Humana Press, Totowa, NJ, 2014, pp. 273-313.

[18] K Tokuyasu and E Yamada, J Cell Biol 6(2) (1959) 225-30.

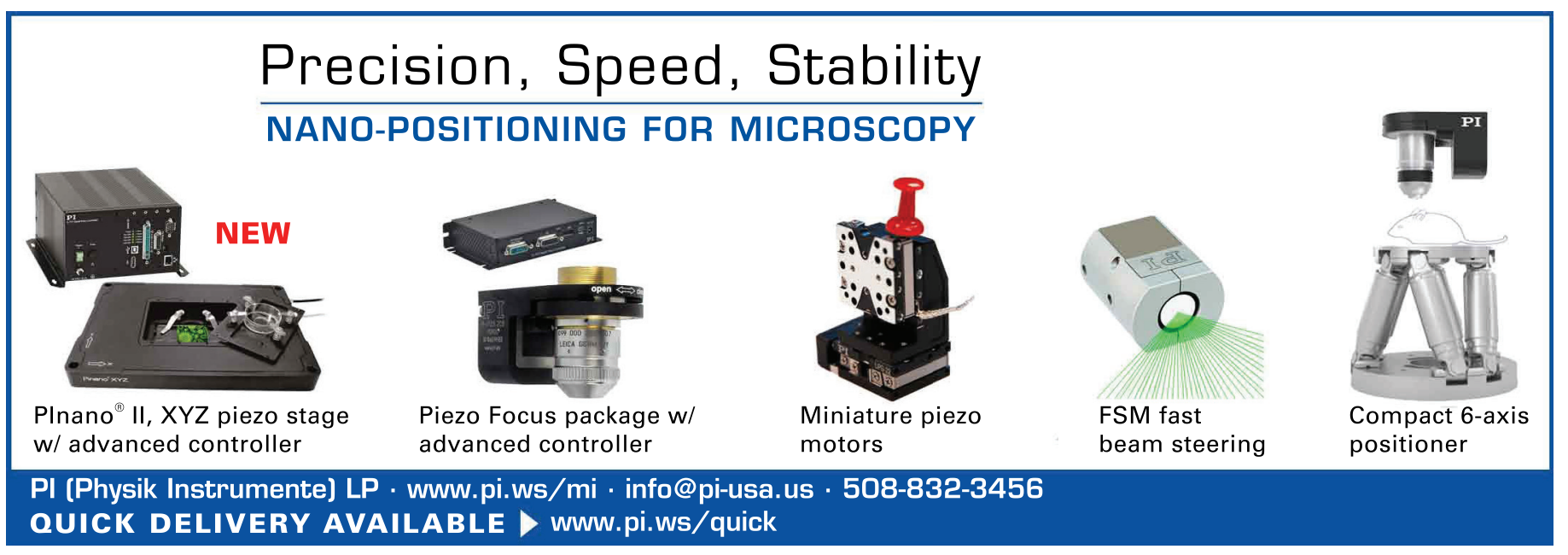




\section{(D) NAVITAR}

$>$ Integrated lens and camera solutions

$>$ Active lens/sensor alignment

$>$ Microscope objectives

$>$ Microscopy cameras



Pi/elink A NAVITAR COMPANY
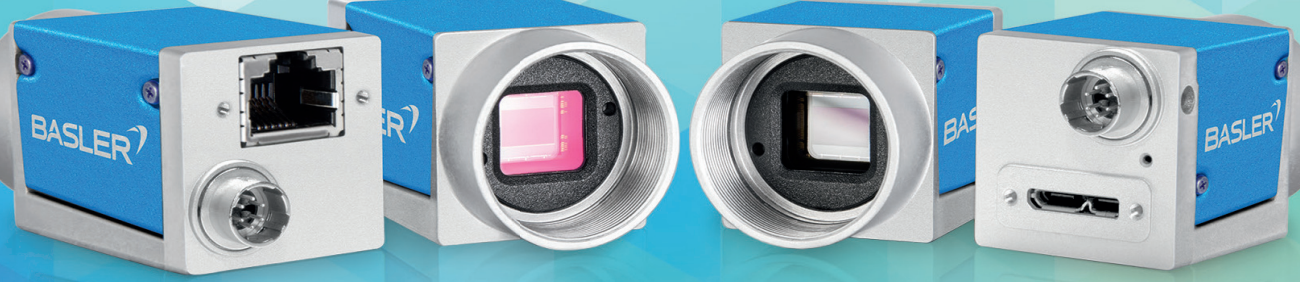

BYE-BYE, CCD SENSORS. HELLO BASLER CMOS CAMERAS.

START A NEW ERA AND SWITCH TO BASLER CAMERAS FOR MEDICAL \& LIFE SCIENCES WITH CMOS SENSOR TECHNOLOGY AT ITS BEST.

With Basler's MED ace, you can be sure to always get the most pristine and colorful images from a precise, stable and utterly dependable camera. Rely on star performers like Sony's Pregius and ON Semiconductor's PYTHON sensors with up to 164 frames per second and 20 MP in resolution. With DIN EN ISO 13485:2016 compliance, you benefit from both a great medical device component and reduced efforts for audits and documentation - that's how we contribute to your quick time-to-market. And the best part: it comes with an outstanding price/ performance ratio.

Be prepared for Sony's discontinuation of CCD sensor technology with us as experienced partner for robust and easy-to-work-with cameras. Take the leap with Basler CMOS cameras for Medical \& Life Sciences.

Learn more at baslerweb.com/MEDICAL.

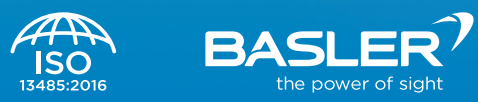




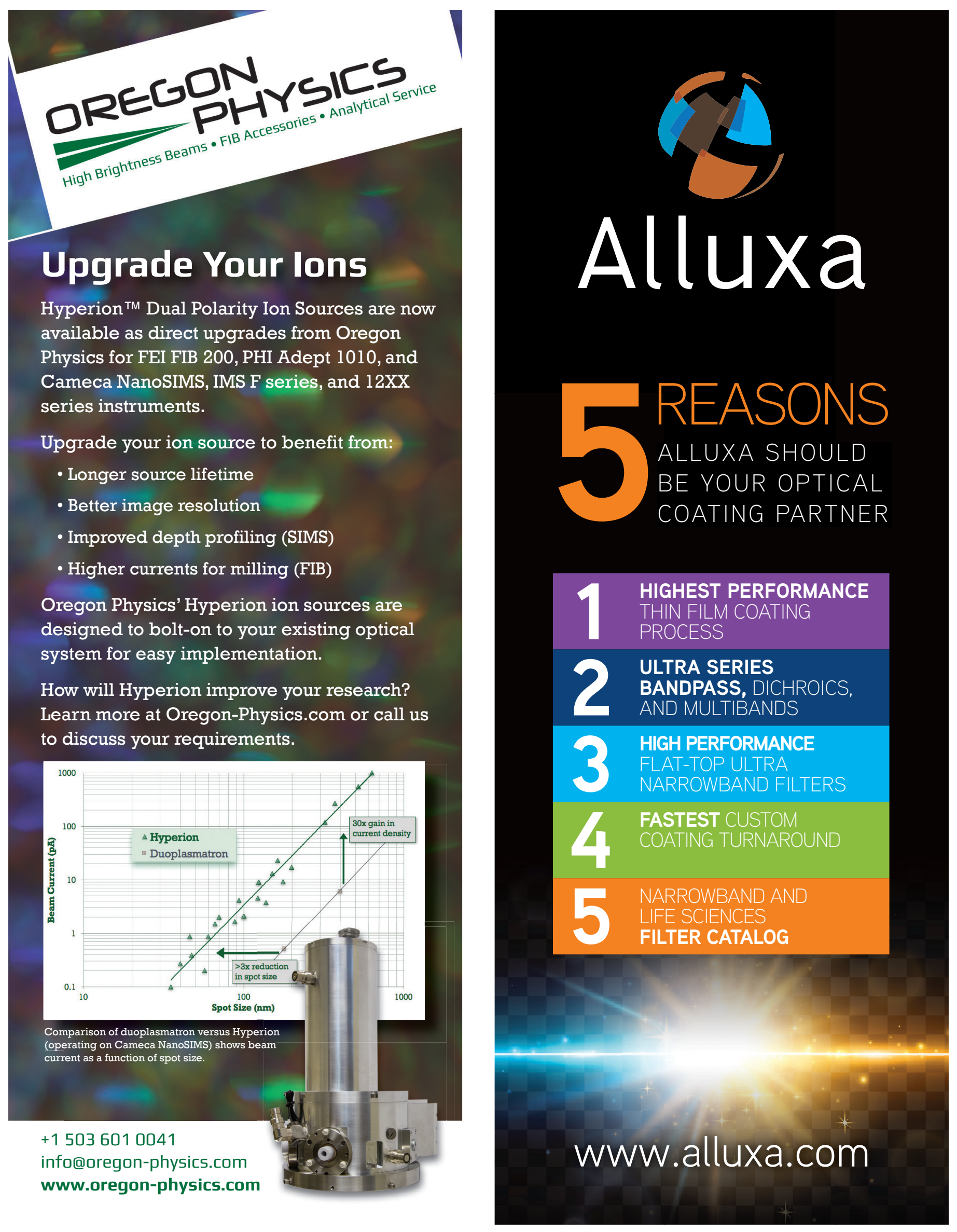

